\title{
Stress Urinary Incontinence post-Holmium Laser Enucleation of the Prostate: a Single-Surgeon Experience
}

Akhil K. Das ${ }^{1}$, Seth Teplitsky ${ }^{1}$, Thenappan Chandrasekar ${ }^{1}$, Tomy Perez ${ }^{1}$, Jenny Guo ${ }^{1}$, Joon Yau Leong ${ }^{1}$, Patrick J. Shenot ${ }^{1}$

${ }^{1}$ Department of Urology, Sidney Kimmel Medical College at Thomas Jefferson University, Philadelphia, $P A, U S A$

\section{ABSTRACT}

Purpose: To identify incidence and predictors of stress urinary incontinence (SUI) following Holmium laser enucleation of the prostate (HoLEP).

Materials and Methods: We performed a retrospective review of 589 HoLEP patients from 2012-2018. Patients were assessed at pre-operative and post-operative visits. Univariate and multivariate regression analyses were performed to identify predictors of SUI.

Results: 52/589 patients (8.8\%) developed transient SUI, while 9/589 (1.5\%) developed long-term SUI. tSUI resolved for 46 patients (88.5\%) within the first six weeks and in 6 patients (11.5\%) between 6 weeks to 3 months. Long-term SUI patients required intervention, achieving continence at 16.4 months on average, 44 men (70.9\%) with incontinence were catheter dependent preoperatively. Mean prostatic volume was $148.7 \mathrm{~mL}$ in tSUI patients, $111.6 \mathrm{~mL}$ in long-term SUI, and $87.9 \mathrm{~mL}$ in others $(\mathrm{p}<0.0001)$. On univariate analysis, laser energy used $(p<0.0001)$, laser "on" time $(p=0.0204)$, resected prostate weight $(\mathrm{p}<0.0001)$, overall International Prostate Symptom Score (IPSS) $(p=0.0005)$, and IPSS QOL $(p=0.02)$ were associated with SUI. On multivariate analysis, resected prostate weight was predictive of any SUI and tSUI, with no risk factors identified for long-term SUI.

Conclusion: Post-HoLEP SUI occurs in $~ 10 \%$ of patients, with $1.5 \%$ continuing beyond six months. Most patients with tSUI recover within the first six weeks. Prostate size $>100 \mathrm{~g}$ and catheter dependency are associated with increased risk tSUI. Larger prostate volume is an independent predictor of any SUI, and tSUI.

\section{ARTICLE INFO}

(D) Seth Teplitsky
http://orcid.org/0000-0001-9242-1360
Keywords:
Epidemiology; physiopathology
[Subheading]; Urinary
Incontinence

Int Braz J Urol. 2020; 46: 624-31

Submitted for publication:

June 22, 2019

Accepted after revision:

August 30, 2019

Published as Ahead of Print:

March 25, 2020

\section{INTRODUCTION}

Benign prostatic hyperplasia $(\mathrm{BPH})$ is a common condition affecting many older men. In the United States, more than $70 \%$ of men aged 60-69 years have symptoms associated with BPH. Currently, lower urinary tract symptoms (LUTS) impact almost $80 \%$ of men older than 70 (1).
Historically, transurethral resection of the prostate (TURP) has been the gold standard for endoscopic management of BPH (2). This technique, although effective, has many potential adverse effects and limitations which have prompted the advent of newer treatment modalities for BPH (3-5). Holmium-laser enucleation of the prostate (HoLEP) is one of the most prominent newer modalities. HoLEP is size independent and can be 
used for enucleation of prostates over $100 \mathrm{~g}$, which has traditionally been a limitation of TURP. Recent studies have shown that HoLEP is equally as effective, and potentially more effective, when compared to TURP and open simple prostatectomy across a variety of outcomes (4).

With HoLEP now recognized in the AUA guidelines as a viable alternative treatment option for those with moderate to severe LUTS, it is important to understand better the adverse event profile associated with this procedure (6). In our institutional experience, the most common complication encountered with HoLEP is postoperative stress urinary incontinence (SUI). The vast majority of SUI seen after HoLEP is transient, with the majority of cases resolving within one year (7). We have found that transient SUI (tSUI) represents one of the most common complaints affecting patient satisfaction and the quality of life postoperatively. Recent reports note that the rates of postoperative SUI range between 1.4\% and 44\% following HoLEP (8-11). The wide range in reported SUI rates is most likely multifactorial and may be due to different operative techniques, surgeon experience, and patient-specific factors. Unfortunately, many of these studies are limited by small sample sizes and technique heterogeneity.

With this study, we aim to more accurately define SUI rates using a large single-surgeon single-institutional experience and to identify the incidence and predictors of SUI following HoLEP. With this knowledge, surgeons can better counsel patients regarding the procedure allowing for more informed patient decision making and improve patient satisfaction.

\section{MATERIALS AND METHODS}

We performed an IRB approved (Control \#12D.50) retrospective chart review of all patients undergoing HoLEP at our institution between January 2012 and June 2018. Our review included the charts of all patient who underwent HoLEP at our institution within this time period under the care of a single surgeon. The exclusion criteria for this review include incomplete surgical resection and lack of post-operative follow-up. Baseline demographic data collected included age, body mass index (BMI), serum prostate-specific antigen (PSA), prostate volume, peak Uroflow rate, mean Uroflow rate, PVR volume, IPSS score, and IPSS QOL rating. All patients underwent urodynamic testing (Laborie Medical Technologies ${ }^{\circledR}$ ) before undergoing surgery to confirm the diagnosis of bladder outlet obstruction. All procedures were performed by a single experienced surgeon (A.D.) who had performed more than one thousand HoLEP cases before the study period. Postoperative clinic visits were conducted within two weeks, at six weeks, and at three months. Assessment at postoperative visits included the IPSS questionnaire, PVR, and Uroflow testing.

Our trilobar HoLEP technique has been described previously, but in brief, a 26 French (Fr) continuous flow resectoscope with a laser bridge adapter and an endoscopic camera are utilized (12). The laser fiber is passed through a 6Fr open-ended ureteral catheter. A 100 Watt holmium laser with an end-firing 550-micron laser fiber is used with energy settings of $2.0 \mathrm{~J}$ and $50 \mathrm{~Hz}$. After trilobar enucleation is completed, a morcellator, grasper, or both are used to clear the bladder of any prostatic tissue.

At postoperative visits, SUI was assessed clinically, which was defined as incontinence during activity with a patient-reported negative impact on quality of life. Any patient who reported incontinence which necessitated the utilization of undergarment pads or diapers was considered "incontinence", while those who did not report needing any pads or diapers were considered continent for our study purposes. SUI was differentiated from other forms of incontinence by careful history taking and physical examination in the clinic. Patients with different types of incontinence, such as urge or mixed, were excluded from this study. For study purposes, SUI was considered transient if it resolved within six months of the procedure date, in following with previous literature (6). Any leakage beyond six months was deemed to be long-term SUI.

Additional risk factors assessed included prostate size as measured by TRUS, CT, or MRI imaging. Patients were risk stratified based on preoperative prostate size $(>100 \mathrm{~g}$ or $\leq 100 \mathrm{~g})$ and pre-operative catheter dependency status (clean intermittent catheterization and continuous urethral drainage). 
Univariate analysis for baseline demographics and perioperative risk factors were completed using the chi-square test for categorical variables and ANOVA for comparison of continuous variables. Multivariable logistic regression was completed to identify factors predictive of increased risk for any SUI, transient SUI, and long-term SUI after HoLEP. Significant factors from the univariate analysis were included in the multivariable analysis. Analyses were completed using SPSS ${ }^{\circledR}$, version 23.0.

\section{RESULTS}

Five hundred eighty nine men undergoing HoLEP during the study period were identified. Postoperative tSUI occurred in 52 men (8.8\%), while 9 (1.5\%) had long-term SUI, for a total of $61(10.4 \%)$ patients who experienced any SUI after HoLEP. Of the patients who experienced tSUI, all had their incontinence resolved within three months. 46 men (88.5\%) with tSUI had full resolution of incontinence within the first six weeks, while the remaining 6 men (11.5\%) resolved between six weeks and three months.

Table-1 highlights preoperative baseline characteristics as well as perioperative results. Except for pre-operative prostate size (tSUI: $148.7 \pm 56.8 \mathrm{~mL}$, long-term SUI: 98.0 $\pm 50.1 \mathrm{~mL}$, no SUI: $92.2 \pm 50.6 \mathrm{~mL}, \mathrm{p}<0.0001$ ),

Table 1 - Baseline Characteristics, Preoperative, and Perioperative Data.

\begin{tabular}{|c|c|c|c|c|}
\hline & $\begin{array}{l}\text { Patients with no } \\
\text { SUI }(n=528)\end{array}$ & tSUI patients $(n=52)$ & Long-term SUI $(n=9)$ & $\mathrm{p}$-value \\
\hline \multicolumn{5}{|l|}{ Preoperative Data } \\
\hline Age & $70.6 \pm 8.5$ & $72.0 \pm 8.9$ & $65.6 \pm 5.7$ & 0.1027 \\
\hline BMI & $28.7 \pm 7.8$ & $29.6 \pm 5.8$ & $28.2 \pm 4.1$ & 0.7026 \\
\hline Serum PSA (ng/mL) & $10.03 \pm 47.45$ & $6.7 \pm 7.4$ & $9.0 \pm 7.1$ & 0.9388 \\
\hline Prostate Size $(\mathrm{mL})$ & $92.2 \pm 50.6$ & $148.7 \pm 56.8$ & $111.6 \pm 48.5$ & $<0.0001$ \\
\hline Pre-0p Uroflow Peak Flow (mL/s) & $8.6 \pm 9.9$ & $14.7 \pm 23.5$ & $11.5 \pm 10.8$ & 0.0009 \\
\hline Pre-Op Uroflow Mean Flow (mL/s) & $3.4 \pm 2.5$ & $3.7 \pm 3.2$ & $3.5 \pm 1.8$ & 0.9367 \\
\hline Pre-0p Post Void Residual (mL) & $238.8 \pm 249.2$ & $297.0 \pm 298.1$ & $185.2 \pm 112.3$ & 0.3030 \\
\hline Pre-Op IPSS Results & $19.7 \pm 8.5$ & $17.9 \pm 9.8$ & $19.4 \pm 5.8$ & 0.5897 \\
\hline Pre-Op IPSS QOL Results & $3.6 \pm 1.2$ & $3.5 \pm 1.5$ & $3.8 \pm 0.7$ & 0.8231 \\
\hline Pre-operative Catheterization (N, \%) & $201(38.1 \%)$ & $37(71.2 \%)$ & $7(77.8 \%)$ & $<0.0001$ \\
\hline \multicolumn{5}{|l|}{ Perioperative Data } \\
\hline Laser Energy Used (kJ) & $339.3 \pm 190.4$ & $514.4 \pm 151.4$ & $434.3 \pm 145.8$ & $<0.0001$ \\
\hline Laser On Time (min) & $118.7 \pm 72.8$ & $163.5 \pm 89.7$ & $174.2 \pm 67.4$ & 0.0204 \\
\hline Resected Prostate Weight (g) & $70.2 \pm 42.8$ & $135.5 \pm 70.5$ & $103.2 \pm 52.4$ & $<0.0001$ \\
\hline Post-Op Catheterization Time (days) & $5.5 \pm 3.5$ & $4.9 \pm 2.1$ & $6.6 \pm 4.6$ & 0.6139 \\
\hline Post-Op Uroflow Peak Flow (mL/s) & $24.3 \pm 17.6$ & $24.0 \pm 11.6$ & $24.8 \pm 9.5$ & 0.9926 \\
\hline Post-0p Uroflow Mean Flow (mL/s) & $6.4 \pm 4.8$ & $5.1 \pm 3.2$ & $6.4 \pm 1.7$ & 0.6384 \\
\hline Post-Op Post Void Residual (mL) & $63.4 \pm 89.3$ & $63.4 \pm 78.5$ & $10.3 \pm 10.5$ & 0.3414 \\
\hline Post-Op IPSS Results & $6.8 \pm 5.9$ & $9.5 \pm 8.3$ & $16.7 \pm 11.4$ & 0.0005 \\
\hline Post-0p IPSS Q0L Results & $1.1 \pm 1.4$ & $1.8 \pm 1.4$ & $2.4 \pm 2.0$ & 0.0214 \\
\hline
\end{tabular}

Continuous data is presented as mean \pm standard deviation; categorical data as proportions 
pre-operative Qmax (tSUI: $14.7 \pm 23.5 \mathrm{~mL} / \mathrm{s}$, long-term SUI: $11.5 \pm 10.8 \mathrm{~mL} / \mathrm{s}$, no SUI: $8.6 \pm 9.9 \mathrm{~mL} / \mathrm{s}$, $\mathrm{p}=0.0009)$ and pre-operative catheter dependence (tSUI: $71.2 \%$ vs. long-term SUI: $77.8 \%$ vs. no SUI: $38.1 \%, p<0.0001$ ), there was no significant difference between men who developed transient SUI, long-term SUI, and those who did not.

With regard to perioperative and postoperative results, patients who developed SUI were found to have greater laser energy used (tSUI: $514.4 \pm 151.4 \mathrm{~kJ}$ vs. long-term SUI: $434.3 \pm 145.8$ $\mathrm{kJ}$ vs. no SUI :339.3 $\pm 190.4 \mathrm{~kJ}, \mathrm{p}<0.0001)$, longer laser "on" time (tSUI: $163.5 \pm 89.7 \mathrm{~min}$, long-term SUI: $174.2 \pm 67.4 \mathrm{~min}$, no SUI: $118.7 \pm 72.8 \mathrm{~min}$, $\mathrm{p}=0.0204$ ), larger resected prostate weight (tSUI: $135.5 \pm 70.5 \mathrm{~g}$, long-term SUI: $103.2 \pm 52.4 \mathrm{~g}$, no SUI: $70.2 \pm 42.8 \mathrm{~g}, \mathrm{p}<0.0001)$, higher overall IPSS score (tSUI: $9.5 \pm 8.3$, long term SUI: $16.7 \pm 11.4$, no SUI: $6.8 \pm 5.9, p=0.0005)$ and IPSS QOL scores (tSUI: $1.8 \pm 1.4$, long term SUI: $2.4 \pm 2.0$, no SUI: $1.1 \pm 1.4, \mathrm{p}=0.0214)$.

When patients with tSUI were stratified by preoperative prostate volume of $>100 \mathrm{~g}(\mathrm{n}=44)$ or $\leq 100 \mathrm{~g}(\mathrm{n}=8), 8$ men $(100 \%)$ with prostates $\leq 100 \mathrm{~g}$ had the resolution of tSUI within 6 weeks. In the 44 men with larger $(>100 \mathrm{~g})$ prostates, $38(86.3 \%)$ had the resolution of tSUI within 6 weeks, while the remaining $6(13.6 \%)$ had the resolution between 6 weeks to 3 months. There was not a statistical significance $(p=0.2394)$ in recovery time when comparing larger prostates $(>100 \mathrm{~g})$ to smaller prostates $(\leq 100 \mathrm{~g})$.

On multivariable logistic regression analysis, we performed multiple analyses looking for predictive factors predisposing patients to any SUI (Supplementary Table-1.1), tSUI (Supplementary Table-1.2), and long-term SUI (Supplementary Table-1.3). Results showed that only resected prostate weight was a significant predictor of developing any SUI (HR 1.020, 95\% CI 1.007-1.033, $\mathrm{p}<0.05$ ) and tSUI (HR 1.019, 95\% CI 1.006-1.032, $\mathrm{p}<0.05)$. There were no risk factors identified for long-term SUI patients.

\section{DISCUSSION}

HoLEP has struggled to gain widespread adoption within the urology community due to a well-established steep learning curve, requiring up to 50 cases to become proficient $(11,13,14)$ The potential development of tSUI has also been a limiting factor in its uptake. SUI, the involuntary leakage of urine, is distressing and has been shown to decrease the quality of life in patients (15). Patient distress likely plays a role in the avoidance of this prostate reducing technique by surgeons.

In our study, we evaluated the incidence and predictors of SUI after HoLEP. We then looked to compare our results to those of other prostate reducing procedures in the literature. In patients undergoing TURP, the incidence of long-term stress incontinence is rare $(\sim 1 \%)$, although $30-40 \%$ of patients have tSUI resolving within six months $(16,17)$. On the other hand, in men undergoing open simple prostatectomy (OP), there is a much higher incidence of long-term incontinence ranging between 1-40\% depending on the technique utilized, with more current data showing $20.2 \%$ of patients becoming permanently incontinent (18-21). Further studies have shown a 38.6\% incidence of tSUI in the three months following simple prostatectomy (22). In comparison, the incidence of long-term and transient SUI in our single-surgeon series is $1.5 \%$ and $8.8 \%$, respectively. Our results suggest improved surgical outcomes compared to the published literature.

Both HoLEP and simple prostatectomy seek to provide complete enucleation of the adenoma. Our HoLEP technique involves endoscopic dissection of the adenoma in a retrograde fashion, from the distal to the proximal attachments. HoLEP allows for distal visualization of the adenoma, compared to the blind approach taken with OP. We feel that this visualization confers an advantage, as visual landmarks allow for the surgeon to avoid damage to the sphincter, and may play a role in lower incontinence rates seen in HoLEP versus OP.

Variable tSUI rates after HoLEP have been reported in the literature. Previous studies have outlined this complication as occurring in anywhere from 1.4-44\% of patients, of which the vast majority recover full continence by one year $(10,11,23)$. A more recent large cohort study from Japan reported a tSUI rate after HoLEP of $16.6 \%$, which is more consistent with our series (8). However, while the extreme variation in reported SUI 
rates postoperatively is likely a function of small sample sizes, variable operative experience, heterogeneous operative techniques, and possible prior bladder dysfunction, our series has the advantage of being a large single-surgeon series. Shah et al. assessed the amount of time required to regain bladder control following HoLEP and found that it took 42.3 days (24). These results are consistent with our study, in which all patients who developed tSUI had complete a recovery by three months. We assessed if a larger prostate size was associated with rate of tSUI recovery. All six patients who took over 6 weeks to recover had large prostate volume, while no patients took over 6 weeks with a low prostate volume. Though the analysis did not show statistical significance $(p=0.2394)$, the absolute numbers suggest that men with larger prostate may have a slightly slower rate of recovery.

This study also sought to uncover risk factors that may predispose patients to SUI, both transient and long-term. There was a statistical difference seen amongst the three groups on analysis for preoperative prostate size, preoperative Qmax, laser “on” time, laser energy used, and resected prostate volume. A novel finding of our study was the association of preoperative catheter dependence with postoperative incontinence. The cause of this correlation remains unknown, but it is possible that patients requiring preoperative catheterization have more severe $\mathrm{BPH}$, and therefore require longer endoscopic manipulation, which predisposes the patients to SUI. The only prior study looking at risk factors by Nam et al. identified increasing age and operative time as risk factors (25). However, on our multivariate logistic regression analysis, only resected prostate weight was a significant predictor of developing any SUI (HR $1.020,95 \%$ CI 1.007-1.033, $\mathrm{p}<0.05)$ and tSUI (HR 1.019, 95\% CI 1.006-1.032, p <0.05).

Our study showed the rate of long-term incontinence to be very low. While no preoperative risk factors predisposing patients to long-term SUI were identified on multivariate regression, post-analysis chart review of these 9 patients uncovered a high rate of neurological comorbidities. Further investigation showed that 8/9 (88.9\%) patients had a significant neurological history. However, as we cannot accurately capture the incidence of neurological comorbidities that exist within the rest of the population, a comparison was not possible. Of the 8,7 had spinal pathology, including spinal stenosis and degenerative disc disease, while 1 had myasthenia gravis. Critically, all of these patients were able to achieve either complete resolution of SUI or reduction to less than 2 pads/day. The management strategies for these patients included initial pelvic floor exercise therapy, and in those still unsatisfied, coaptite injections. Multiple coaptite injections were given to patients who had partial responses. Only one patient went on to require AUS implantation. These 9 patients achieved a satisfactory level of continence at an average of 16.4 months after the completion of their HoLEP.

When assessing postoperative QOL outcomes, we found that men with tSUI and long-term SUI had worse postoperative IPSS scores (both worse subjective symptoms and quality of life responses) when compared to those patients who did not experience tSUI. As expected, SUI had a substantial negative impact on quality of life. This result is in line with previous reports showing the quality of life impact that incontinence can have on patients (26).

One possible cause of incontinence seen after surgery is sphincter dysfunction, which is likely the result of prolonged endoscopic manipulation. Endoscopic procedures are thought to cause trauma directly to the sphincter, leading to this dysfunction. This dysfunction is thought to be temporary, causing the transient nature of the SUI $(8,11,27)$ A larger prostate size leads to longer operative times, which may explain the correlation between tSUI and both larger prostates and longer operative times. The longer laser time and laser energy used, as well as the heavier weight of resected prostate tissue, are all associated with a larger prostate. All of our results seem to indicate that larger prostates cause longer operations, and therefore increased endoscopic manipulation and risk for tSUI via sphincter dysfunction. As many of the variables associated with tSUI on univariate analysis were surrogate markers of prostate size, this may explain why 
they were not independently associated with tSUI on multivariate regression analysis.

This study is not devoid of limitations, including those inherent to a retrospective study. SUI characterization was primarily dependent on physician documentation. Missing data, especially from operative variables, also limited the robustness of the multivariable analysis. Comorbidities, such as diabetes, that may have been associated with SUI development were not adequately captured.

Regardless of these limitations, this study still represents the largest single-surgeon experience with HoLEP and provided valuable data regarding the incidence, time course and predictors of post-operative tSUI. Transient SUI after HoLEP has notable implications for patient quality of life, which may contribute to the hesitancy in the widespread adoption of HoLEP. Our study demonstrates this tSUI resolves in the majority of patients, usually within the first six weeks. Furthermore, by identifying risk factors that predispose patients to tSUI, preoperative counseling can be enhanced, thus mitigating possible patient frustration and improving both patient and physician satisfaction. This may also provide data for better patient selection in order to avoid these complications after the HoLEP procedure. We feel this study shows the need for future prospective trials and further inquiry into ways to prevent this complication.

\section{CONCLUSIONS}

Overall, our study helps to highlight the relatively low rate of incontinence (10.4\%) seen after HoLEP from an experienced surgeon, with $8.8 \%$ being transient and 1.5\% being long-term. Our results suggest that the overwhelming majority of patients with tSUI recover within the first 6 weeks following HOLEP. Prostate size greater than $100 \mathrm{~g}$ and catheter dependency are associated with increased risk of developing tSUI, and larger prostate volume is an independent predictor of tSUI. While long-term SUI was rare (1.5\%), it is possible that neurologic complications may be a contributing factor, though this requires further study. Those with long-term SUI are most often able to achieve continence with further intervention.

\section{ABBREVIATIONS}

SUI $=$ Stress urinary incontinence tSUI $=$ Transient stress urinary incontinence HoLEP = Holmium laser enucleation of prostate $\mathrm{BPH}=$ Benign prostatic hyperplasia

LUTS $=$ Lower urinary tract symptoms TURP $=$ Transurethral resection of prostate

PVR $=$ Post-void residual

$\mathrm{OP}=$ Open prostatectomy

Qmax = Peak flow

IPSS = International prostate symptom score

QOL $=$ Quality of life

PSA $=$ Prostate-specific antigen

$\mathrm{Fr}=$ French

\section{CONFLICT OF INTEREST}

None declared.

\section{REFERENCES}

1. Wei JT, Calhoun E, Jacobsen SJ. Urologic diseases in America project: benign prostatic hyperplasia. J Urol. 2005;173:1256-61.

2. McVary KT, Roehrborn CG, Avins AL, Barry MJ, Bruskewitz RC, Donnell RF, et al. Update on AUA guideline on the management of benign prostatic hyperplasia. J Urol. 2011;185:1793-803.

3. Xie CY, Zhu GB, Wang XH, Liu XB. Five-year follow-up results of a randomized controlled trial comparing bipolar plasmakinetic and monopolar transurethral resection of the prostate. Yonsei Med J. 2012;53:734-41.

4. Chung DE, Te AE. New techniques for laser prostatectomy: an update. Ther Adv Urol. 2009;1:85-97.

5. Zarrabi A, Gross AJ. The evolution of lasers in urology. Ther Adv Urol. 2011;3:81-9.

6. Foster HE, Barry MJ, Dahm P, Gandhi MC, Kaplan SA, Kohler TS, et al. Surgical Management of Lower Urinary Tract Symptoms Attributed to Benign Prostatic Hyperplasia: AUA Guideline. J Urol. 2018;200:612-9.

7. Shiraishi Y, Yoshimura K, Inoue T, Okubo K, Watanabe J, Kamba $\mathrm{T}$, et al. [One year follow-up results of urinary incontinence and sexual function after holmium laser enucleation of the prostate]. Hinyokika Kiyo. 2009;55:539-43.

8. Endo F, Shiga Y, Minagawa S, Iwabuchi T, Fujisaki A, Yashi $M$, et al. Anteroposterior dissection HoLEP: a modification to prevent transient stress urinary incontinence. Urology. 2010;76:1451-5. 
9. Kuntz RM, Ahyai S, Lehrich K, Fayad A. Transurethral holmium laser enucleation of the prostate versus transurethral electrocautery resection of the prostate: a randomized prospective trial in 200 patients. J Urol. 2004;172:1012-6.

10. Montorsi F, Naspro R, Salonia A, Suardi N, Briganti A, Zanoni $M$, et al. Holmium laser enucleation versus transurethral resection of the prostate: results from a 2-center, prospective, randomized trial in patients with obstructive benign prostatic hyperplasia. J Urol. 2004;172(5 Pt 1):1926-9.

11. Seki N, Mochida O, Kinukawa N, Sagiyama K, Naito S. Holmium laser enucleation for prostatic adenoma: analysis of learning curve over the course of 70 consecutive cases. J Urol. 2003;170:1847-50.

12. Kelly DC, Das A. Holmium laser enucleation of the prostate technique for benign prostatic hyperplasia. Can J Urol. 2012;19:6131-4.

13. Shah HN, Mahajan AP, Sodha HS, Hegde S, Mohile PD, Bansal MB. Prospective evaluation of the learning curve for holmium laser enucleation of the prostate. J Urol. 2007;177:1468-74.

14. El-Hakim A, Elhilali MM. Holmium laser enucleation of the prostate can be taught: the first learning experience. BJU Int. 2002;90:863-9.

15. Herr HW. Quality of life of incontinent men after radical prostatectomy. J Urol. 1994;151:652-4.

16. Buckley BS, Lapitan MC; Epidemiology Committee of the Fourth International Consultation on Incontinence, Paris, 2008. Prevalence of urinary incontinence in men, women, and children--current evidence: findings of the Fourth International Consultation on Incontinence. Urology. 2010;76:265-70.

17. Rassweiler J, Teber D, Kuntz R, Hofmann R. Complications of transurethral resection of the prostate (TURP)--incidence, management, and prevention. Eur Urol. 2006;50:969-79.

18. Rodriguez E Jr, Skarecky DW, Ahlering TE. Post-robotic prostatectomy urinary continence: characterization of perfect continence versus occasional dribbling in pad-free men. Urology. 2006;67:785-8.

19. Krupski TL, Saigal CS, Litwin MS. Variation in continence and potency by definition. J Urol. 2003;170(4 Pt 1):1291-4.

20. Olsson LE, Salomon L, Nadu A, Hoznek A, Cicco A, Saint F, et al. Prospective patient-reported continence after laparoscopic radical prostatectomy. Urology. 2001;58:570-2.
21. Haglind E, Carlsson S, Stranne J, Wallerstedt A, Wilderäng $U$, Thorsteinsdottir $T$, et al. Urinary Incontinence and Erectile Dysfunction After Robotic Versus Open Radical Prostatectomy: A Prospective, Controlled, Nonrandomised Trial. Eur Urol. 2015;68:216-25.

22. Naspro R, Suardi N, Salonia A, Scattoni V, Guazzoni G, Colombo $\mathrm{R}$, et al. Holmium laser enucleation of the prostate versus open prostatectomy for prostates $>70 \mathrm{~g}$ : 24-month follow-up. Eur Urol. 2006;50:563-8.

23. Kuntz RM, Lehrich K, Ahyai S. Does perioperative outcome of transurethral holmium laser enucleation of the prostate depend on prostate size? J Endourol. 2004;18:183-8.

24. Shah HN, Mahajan AP, Hegde SS, Bansal MB. Peri-operative complications of olmium laser enucleation of the prostate: experience in the first 280 patients, and a review of literature. BJU Int. 2007;100:94-101.

25. Nam JK, Kim HW, Lee DH, Han JY, Lee JZ, Park SW. Risk Factors for Transient Urinary Incontinence after Holmium Laser Enucleation of the Prostate. World J Mens Health. 2015;33:88-94.

26. Lee YJ, Oh SA, Kim SH, Oh SJ. Patient satisfaction after holmium laser enucleation of the prostate (HoLEP): A prospective cohort study. PLoS One. 2017;12:e0182230.

27. Walz J, Burnett AL, Costello AJ, Eastham JA, Graefen M, Guillonneau B, et al. A critical analysis of the current knowledge of surgical anatomy related to optimization of cancer control and preservation of continence and erection in candidates for radical prostatectomy. Eur Urol. 2010;57:179-92.

\section{Correspondence address:}

Akhil K. Das, MD, FACS

Department of Urology

Sidney Kimmel Medical College at Thomas Jefferson

University

1025 Walnut Street

Suite 1112, College Building Philadelphia, PA 19107, USA

Telephone: +1 215 955-6961

E-mail: akhil.das@jefferson.edu 
Supplementary Table 1.1 - Results from multivariable logistic regression analysis assessing for risk factors for any SUI in HoLEP patients.

\begin{tabular}{lcc}
\hline Variables & OR $(95 \% \mathrm{Cl})$ & $\mathrm{p}$ \\
\hline Age (years) & $0.981(0.928-1.037)$ & 0.494 \\
$\mathrm{BMI}\left(\mathrm{Kg} / \mathrm{m}^{2}\right)$ & $0.983(0.923-1.047)$ & 0.597 \\
Prostate Volume $(\mathrm{mL})$ & $1.009(0.997-1.021)$ & 0.133 \\
Laser Energy Used $(\mathrm{Kj})$ & $0.990(0.996-1.003)$ & 0.763 \\
Laser On Time $(\mathrm{min})$ & $0.997(0.990-1.005)$ & 0.463 \\
Resected Prostate Volume $(\mathrm{cc})$ & $1.020(1.007-1.033)$ & 0.002 \\
\hline
\end{tabular}

Supplementary Table 1.2 - Results from multivariable logistic regression analysis assessing for risk factors for tSUI in HoLEP patients.

\begin{tabular}{lcc}
\hline Variables & OR $(95 \% \mathrm{Cl})$ & $\mathrm{p}$ \\
\hline Age (years) & $1.013(0.949-1.083)$ & 0.694 \\
$\mathrm{BMI}\left(\mathrm{Kg} / \mathrm{m}^{2}\right)$ & $0.987(0.925-1.054)$ & 0.697 \\
Prostate Volume $(\mathrm{mL})$ & $1.011(0.997-1.025)$ & 0.113 \\
Laser Energy Used $(\mathrm{Kj})$ & $1.001(0.996-1.006)$ & 0.731 \\
Laser On Time $(\mathrm{min})$ & $0.995(0.986-1.004)$ & 0.259 \\
Resected Prostate Volume (cc) & $1.019(1.006-1.032)$ & 0.004 \\
\hline
\end{tabular}

Supplementary Table 1.3 - Results from multivariable logistic regression analysis assessing for risk factors for longterm SUI in HoLEP patients.

\begin{tabular}{lcc}
\hline Variables & OR $(95 \% \mathrm{Cl})$ & $\mathrm{p}$ \\
\hline Age (years) & $0.915(0.825-1.014)$ & 0.090 \\
BMI $\left(\mathrm{Kg} / \mathrm{m}^{2}\right)$ & $0.964(0.830-1.120)$ & 0.630 \\
Prostate Volume $(\mathrm{mL})$ & $1.002(0.978-1.026)$ & 0.896 \\
Laser Energy Used $(\mathrm{Kj})$ & $0.997(0.990-1.003)$ & 0.345 \\
Laser On Time (min) & $1.006(0.994-1.019)$ & 0.308 \\
Resected Prostate Volume $(\mathrm{cc})$ & $1.009(0.985-1.034)$ & 0.456 \\
\hline
\end{tabular}

